\title{
Tinea capitis presented as unilateral facial erythematous patches
}

\section{Asmae Abdelmouttalib, Soumaya Hamich, Mariame Meziane, Nadia Ismaili, Laila Benzekri, Karima Senouci}

\author{
Dermatology and Venereology Department, Mohamed V University in Rabat, Morocco
}

Corresponding author: Asmae Abdelmouttalib, MD, E-mail: abdelmouttalibasmae@gmail.com

Tinea capitis is an infection of the hair due to keratinophilic fungi, known as dermatophytes. Although the disease is common in children, several studies have also shown that it is far from unusual in adults, especially in immunocompromised persons [1]. A 31-years-old woman presented with a 3-months history of erythematous and itchy patches on left cheek and auricle. Her pas medical history was significant for systemic lupus erythematosus diagnosed the year before with malar rash, arthralgia, and lupus nephropathy. She was receiving Hydroxychloroquine, low dose prednisone and Cyclophosphamide boluses. Her lupus disease was well controlled and the facial lesions did not regress in spite of a well-respected sun protection including appropriate clothing, sunscreens, and behavior change. Therefore, she was referred to the dermatology department. Clinical examination showed a malar rash more accentuated on the left side; and erythematous and squamous patches on temporal hairline extended to the left auricle, the cheek and the nasolabial folds (Figs. 1 and 2). Dermoscopic examination showed erythema and follicular keratosis with broken and corkscrew hairs (Fig. 3). Trichophyton tinea capitis was suspected, a mycological test with direct examination and culture confirmed this diagnosis. A treatment with oral terbinafine was started at a dose of $4 \mathrm{mg} / \mathrm{kg} /$ day for a period of six weeks with favorable results.

Secretion of sebum and colonization by Pityrosporum orbiculareare supported to protect the scalp against dermatophytic invasion after puberty, but an immune defect may also facilitate hair invasion [2]. Because of a low index of suspicion for tinea capitis in adults

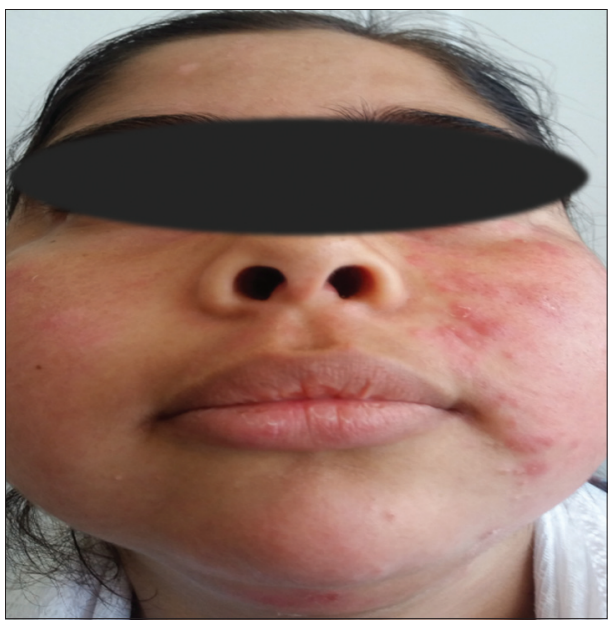

Figure 1: Erythematous and squamous patches on the left cheek and the nasolabial folds.

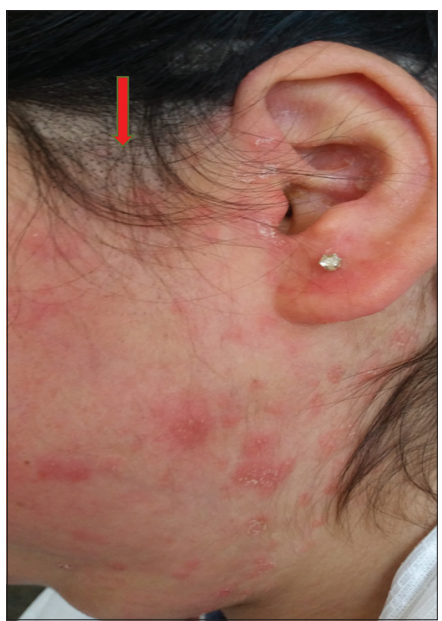

Figure 2: Scaling on the left auricle and temporal hairline with broken hairs (red arrow).

with scaling and alopecia, diagnosis and appropriate treatment are often delayed.

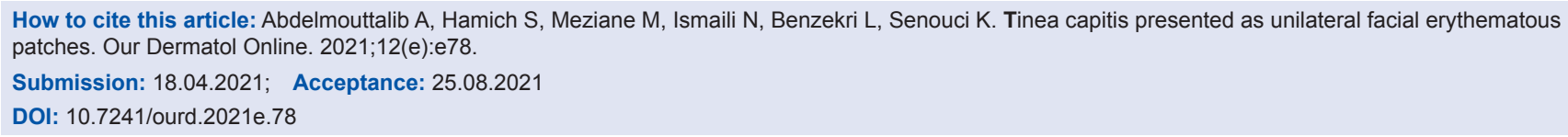


www.odermatol.com

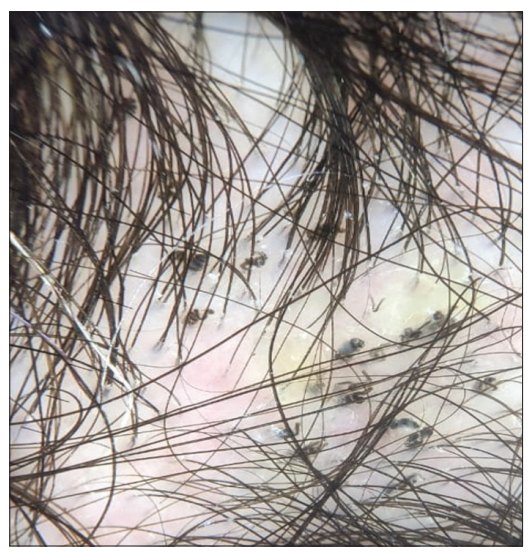

Figure 3: Dermoscopic examination showing follicular keratosis with broken and corkscrew hairs.

\section{Consent}

The examination of the patient was conducted according to the principles of the Declaration of Helsinki.
The authors certify that they have obtained all appropriate patient consent forms, in which the patients gave their consent for images and other clinical information to be included in the journal. The patients understand that their names and initials will not be published and due effort will be made to conceal their identity, but that anonymity cannot be guaranteed.

\section{REFERENCES}

1. Ely JW, Rosenfeld S, Seabury Stone M. Diagnosis and management of tinea infections. Am Fam Physician. 2014;90:702-10.

2. Torres - Guerrero E, Espinoza - Hernández CJ, Arroyo Camarena S, Atoche-Diéguez CE. Tinea caused by Microsporum gypseum. Our Dermatol Online. 2018;9:380-5.

Copyright by Asmae Abdelmouttalib, et al. This is an open access article distributed under the terms of the Creative Commons Attribution License, which permits unrestricted use, distribution, and reproduction in any medium, provided the original author and source are credited.

Source of Support: Nil, Conflict of Interest: None declared. 